\title{
Complexity and plasticity of cardiac cellular composition
}

The cellular composition of the heart is highly heterogeneous, plastic and sex-specific according to two papers published in Nature and Cardiovascular Research. These findings have important implications for understanding the developmental origins and functional specialization of the heart and also highlight the importance of considering biological sex in studies examining cardiac physiology, stress responses and therapeutic strategies.

In an article published in Nature, researchers isolated approximately half a million single cells and nuclei from 14 donor human hearts and analysed them using single-cell RNA sequencing, single-nucleus RNA sequencing and multiplex RNA small-molecule fluorescence in situ hybridization.

Atrial and ventricular tissues differed in their cellular composition. Atrial tissues contained $30.1 \%$ cardiomyocytes, $24.3 \%$ fibroblasts, $17.1 \%$ mural cells (pericytes and vascular smooth muscle cells), $12.2 \%$ endothelial cells and $10.4 \%$ immune cells (myeloid and lymphoid cells). By contrast, ventricular tissues contained $49.2 \%$ cardiomyocytes, $21.2 \%$ mural cells, $15.5 \%$ fibroblasts, $7.8 \%$ endothelial cells and 5.3\% immune cells. Furthermore, atrial and ventricular cardiomyocytes had strikingly distinct transcriptional signatures, indicative of their different developmental origins and electrophysiological, contractile and secretory properties.

In the vascular compartment, 17 distinct cell populations with anatomical and arterio-venous specificities were identified. These included ten populations of endothelial cell, four clusters of pericyte, two groups of vascular smooth muscle cell and a small, distinct population of mesothelial cells.

The researchers also identified 7 populations of cardiac fibroblast, 21 states of cardiac immune cell (8 populations of lymphoid cell and 13 populations of myeloid cell, including multiple macrophage, monocyte and dendritic cell subtypes), 6 clusters of neuronal cell and 3 categories of adipocyte.

The investigators note that the expression levels of $A C E 2$, encoding angiotensin-converting enzyme 2, which is the receptor used by SARSCoV-2 to infect cells, were highest in pericytes, followed by fibroblasts and lowest in cardiomyocytes (with twofold higher expression in ventricular cardiomyocytes than in atrial cardiomyocytes).

In ventricular tissues, the proportion of cardiomyocytes was higher in women than in

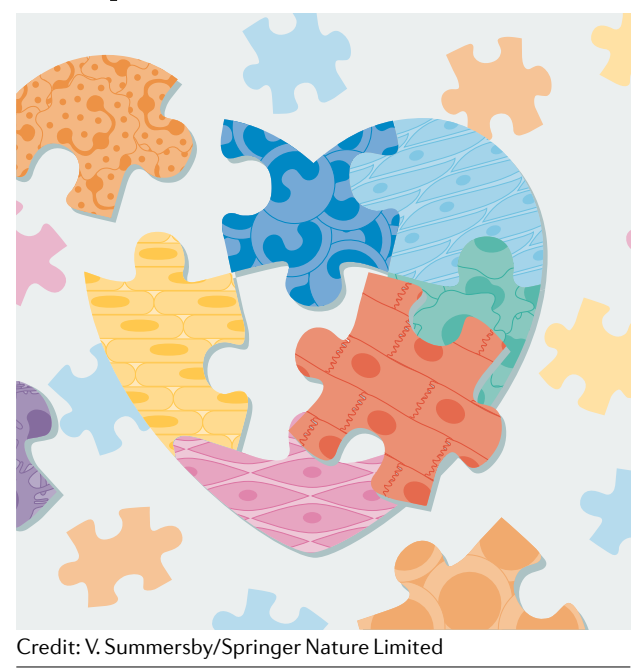

men; otherwise, the researchers did not note significant differences in cellular distributions between male and female hearts. However, in another paper published in Cardiovascular Research, Alexander Pinto and colleagues investigated the sex-specific differences in the non-myocyte composition of mouse hearts. The researchers found higher levels of resident mesenchymal cells in female hearts than in male hearts. In addition, female hearts had higher proportions of natural killer cells and T cells, whereas male hearts had higher proportions of total myeloid cells, granulocytes and B cells.

These sex-specific differences develop soon after birth and are controlled by gonadal hormones. Gonadectomy caused rapid changes in cardiac cellular composition in the mice, and many of these changes were reversed by the reintroduction of oestrogen or testosterone to female and male mice, respectively, indicating that the cellular composition of the heart is plastic and is regulated by endocrine factors.

"The heart not only has a diverse array of cells - beyond cardiomyocytes and fibroblasts - but also has a sex-specific profile," comments Pinto. "However, much biomedical research is calibrated to address disease phenotypes observed in males only. This is concerning, particularly in the context of preclinical drug development and testing," he cautions.

Gregory B. Lim

ORIGINAL ARTICLES Litviňuková, M. et al. Cells of the adult human heart. Nature https://doi.org/10.1038/s41586-020-2797-4 (2020)| Squiers, G. T. et al. Cardiac cellularity is dependent upon biological sex and is regulated by gonadal hormones. Cardiovasc. Res. https:// doi.org/10.1093/cvr/cvaa265 (2020)

\section{ACE2 level as a marker of CVD}

Higher levels of angiotensinconverting enzyme 2 (ACE2) in plasma are associated with a greater risk of major cardiovascular disease (CVD) events, according to a global, population-based study. ACE2 is a component of the counter-regulatory renin-angiotensin system, which has important roles in various CVDs. Moreover, ACE2 is the receptor used by SARS-CoV-2, the virus that causes COVID-19, to enter host cells. Therefore, understanding the function and prognostic role of ACE2 is of increasing importance.

A study including 10,753 participants from the PURE study, which enrolled participants from 14 countries across five continents, now shows that high ACE2 levels in plasma are associated with an increased risk of all-cause death as well as cardiovascular and noncardiovascular deaths. Plasma ACE2 levels were also associated with a higher risk of incident heart failure, myocardial infarction, stroke and diabetes mellitus, independently of age, sex, ancestry and traditional risk factors for CVD. Except for incident heart failure, all associations remained significant after adjusting for B-type natriuretic peptide levels. Moreover, plasma ACE2 level was the highestranked predictor of death compared with established risk factors for CVD (smoking, diabetes, blood pressure, blood lipids and BMI) and was a better predictor of heart failure, stroke and myocardial infarction than several of the established risk factors. Men had higher plasma ACE2 levels than women; ACE2 levels varied by ancestry; and higher BMI, older age, diabetes, higher blood pressure, higher LDL-cholesterol level and smoking were associated with increased plasma ACE2 levels.

Irene Fernández-Ruiz

ORIGINAL ARTICLE Narula, S. et al. Plasma ACE2 and risk of death or cardiometabolic diseases: a case-cohort analysis. Lancet https://doi.org/ 10.1016/S0140-6736(20)31964-4 (2020) RELATED ARTICLE Paz Ocaranza, M. et al. Counter-regulatory renin-angiotensin system in cardiovascular disease. Nat. Rev. Cardiol. 17, 116-129 (2020) 